\title{
Dinámica de la desigualdad regional en México, 1970-1980
}

\author{
Germán Osuna Gastelán*
}

El objetivo de este trabajo es medir la desiguaidad que prevalece entre ios niveles de desarrollo económico y social de las entidades federativas del país y analizar su evolución en el periodo 1970-1980. Para ello, se aplica metodología de estratificación multivariada con objeto de construir grupos de estados con condiciones homogéneas de nivel de vida y se estiman índices de Atkinson para la medición de la desigualdad en el tiempo.

Los resultados obtenidos indican una disminución de las disparidades existentes en la distribución especial del desarrollo durante la década de los setenta y señalan importantes beneficios potenciales derivados de una política redistributiva del producto en el plano territorial.

\section{Introducción}

El desarrollo de un país no se realiza por igual en los diversos núcleos de población que se asientan a lo largo de su territorio. En México prevalecen disparidades sustanciales en los niveles de vida alcanzados por sus regiones. En 1980, por ejemplo, las entidades federativas de mayor desarrollo generaron un producto per cápita promedio de 25 mil pesos, a precios de 1970, el cual triplicó al obtenido por los estados de mayor pobreza. En los extremos del conjunto de entidades, la relación entre el Distrito Federal y Oaxaca fue de cinco a uno.

El objetivo de este trabajo es dimensionar la desigualdad del desarrollo estatal de México y analizar su dinámica durante la década de los años setenta. La investigación aporta el empleo de técnicas estadísticas de creación reciente, de utilidad para el análisis regional. Se aplica metodología de estratificación multivariada para la construcción de grupos de entidades con condiciones homogéneas de nivel de vida y se estiman índices de Atkinson para la medición de la desigualdad en el tiempo. El periodo seleccionado permite ampliar el horizonte del desarrollo regional del país abarcado en estudios emprendidos con anterioridad. México.

* Economista y politólogo. Profesor del Institüto Tecnológico Autónomo de 
El trabajo está dividido en cuatro partes. En la primera se examinan los aspectos metodológicos relacionados con la medición de las disparidades espaciales. En un segundo aparato se analiza la dinámica de los desequilibrios estatales con base en el producto por habitante, como indicador del nivel de desarrollo. En la siguiente sección se amplía esta definición para incluir diversas variables representativas del bienestar social de la población. Finalmente, se presentan las principales conclusiones de la investigación.

\section{La medición de la desigualdad regional}

El estudio del desarrollo intertemporal de unidades territoriales enfrenta tres oroblemas operacionales básicos: la conceptualización del desarrollo, la estratificación de las unidades de análisis y la medición del grado de desigualdad.

La literatura sobre el desarrollo regional mexicano presenta distintos enfoques en el tratamiento de estos aspectos. Unikel y Victoria (1970, p. 292) cuantifican un índice compuesto del nivel de vida estatal a partir del método de componentes principales. Este índice se utiliza para agrupar a las entidades en estratos de desarrollo relativo y medir los cambios ocurridos en la desigualdad entre estados durante el periodo 1940-1960. Más recientemente, Hernández Laos $(1984$, p. 221) mide la evolución de las disparidades existentes en ocho regiones geográficas del país entre 1900 y 1980, a partir de los datos de producto interno bruto por habitante, utilizando diversos índices de dispersión.

En este trabajo se examina la dinámica de la desigualdad regional con base en dos conceptualizaciones distintas del desarrollo. Como primera aproximación, vinculada con lo relativo a la capacidad económica, se utiliza el producto interno bruto per cápita de las entidades federativas. Con la intención de ampliar la definición a fin de reflejar la utilidad social del esfuerzo productivo, se construye un índice compuesto con la técnica de componentes principales a partir de once variables asociadas con el nivel de vida de la población, relativas a las condiciones de ingresos, educación, vivienda y salud. ${ }^{1}$ De esta manera, es posible contrastar los resultados obtenidos con cada una de las definiciones.

\footnotetext{
${ }^{1}$ Para una descripción del método de componentes principales, véase Ram (1982, pp. 227-247).
} 
En relación con las unidades de análisis es importante mencionar que resulta metodológicamente discutible estudiar las desigualdades regionales a nivel de entidad federativa. A manera de ejemplo, debería utilizarse al área metropolitana de la ciudad de México como unidad de análisis en vez de considerar a la totalidad del Distrito Federal y el Estado de México en forma independiente. La disponibilidad de información no permitió estudiar el fenómeno del desarrollo regional con unidades más adecuadas.

En cuanto al índice de bienestar social estimado, éste proporciona una jerarquización de las entidades de acuerdo con su nivel relativo de desarrollo. En estudios anteriores, este indicador se ha utilizado para establecer grupos de estados con condiciones de nivel de vida presumiblemente similares. Con el objeto de contar con un criterio más preciso de selección de intervalos, se emplea una técnica de estratificación multivariada que permite la obtención de estratos óptimos mediante un proceso iterativo, en el que se reasignan observaciones entre grupos de entidades hasta obtener conglomerados de varianza mínima. Los conjuntos de estados resultantes poseen características homogéneas de bienestar social en términos de los once indicadores considerados. ${ }^{2}$

Para la medición del grado de desigualdad del PIB per cápita y de los indicadores del bienestar social, se utilizan los índices de Gini y Atkinson. Al respecto, se señalan a continuación algunas consideraciones metodológicas sobre la medición de la desigualdad relacionadas con la selección de estos índices.

Diversas medidas han sido construidas para captar, en un número, la información sobre la desigualdad de una distribución. En general, estos índices, se clasifican en normativos y positivos, según hagan o no explícita la función de bienestar social en la que se basan. Entre las medidas positivas se encuentran la varianza, el coeficiente de variación y el índice de Gini. Los índices normativos incluyen los coeficientes de Dalton, Atkinson y Sen.

Varios de estos índices han sido empleados en los estudios sobre el desarrollo regional mexicano, sin hacer explícitas las razones por las que se prefirió una u otra medida. En general, existen diversas propiedades deseables que todo índice de desigualdad debería cumplir y que son útiles para discriminar entre las distintas medidas. Estas propiedades son las siguientes: ${ }^{3}$

1. Si el ingreso de cada individuo se incrementa en un monto

${ }^{2}$ El método corresponde a Jarque (1981, pp. 163-169). En el anexo A se describe en forma general.

${ }^{3}$ Para una descripción formal de estos axiomas, véase Sen $(1973$, p. 52). 
constante, las diferencias absolutas de ingreso deben permanecer inalteradas y disminuir en términos relativos. ${ }^{4}$

2. Si todos los ingresos aumentan en la misma proporción, la medida de desigualdad debe mantenerse sin cambio.

3. La desigualdad no varía si se incrementa en la misma proporción el número de personas que percibe cada ingreso.

4. Si se realiza una transferencia de ingresos de una persona de ingreso a una de menor nivel, la desigualdad debe disminuir, siempre y cuando la transferencia no incremente la diferencia de los ingresos en valor absoluto.

5. Si dos individuos intercambian sus posiciones de ingreso, el grado de desigualdad no se altera.

6. La medida de desigualdad varía entre cero y uno, según exista máxima igualdad o desigualdad, respectivamente. ${ }^{5}$ De las distintas medidas positivas de desigualdad comúnmente utilizadas, sólo el índice de Gini satisface estos seis axiomas, de lo cual se deriva su empleo en este trabajo.

El Gini es un coeficiente directamente relacionado con la curva de Lorenz. Dicha curva se define como la línea que une los puntos que relacionan la proporción acumulada de pobla'ción y el porcentaje acumulado de ingreso. Si todos los estratos de la población perciben el mismo ingreso, la curva de Lorenz se convierte en la diagonal. En ausencia de igualdad, la curva se encontrará por debajo de esta línea. Entre más cercana se halle la curva a la diagonal, el grado de desigualdad de la distribución del ingreso será menor.

La gráfica 1 muestra la curva de Lorenz correspondiente a la distribución estatal del producto interno bruto en 1980. El coeficiente de Gini de esta distribución se define como la razón entre el área que se encuentra comprendida entre la línea de igualdad y la curva de Lorenz, y el área del triángulo delimitada por la línea de igualdad y los ejes.

Si las curvas de Lorenz de dos distribuciones no se intersectan, es posible concluir invariablemente que la distribución más cercana a la diagonal es más igualitaria que la otra. En este caso, cualquiera de las medidas comunes de desigualdad registraría el mismo ordenamiento de las distribuciones.

$\mathrm{Si}$, por el contrario, las curvas se cruzan, como acontece en el caso presentado en este artículo (gráfica 2), resulta claro que no es factible arribar a esta conclusión, ya que existen grupos de in-

\footnotetext{
${ }^{4}$ Por ser de uso común, se emplean los términos ingreso e individuo para denotar al atributo y la unidad de análisis, respectivamente.

5 Este último axioma es meramente para facilitar la interpretación del índice.
} 
GRÁFICA 1

Curva de Lorenz del producto Interno bruto estatal 1980 PIB

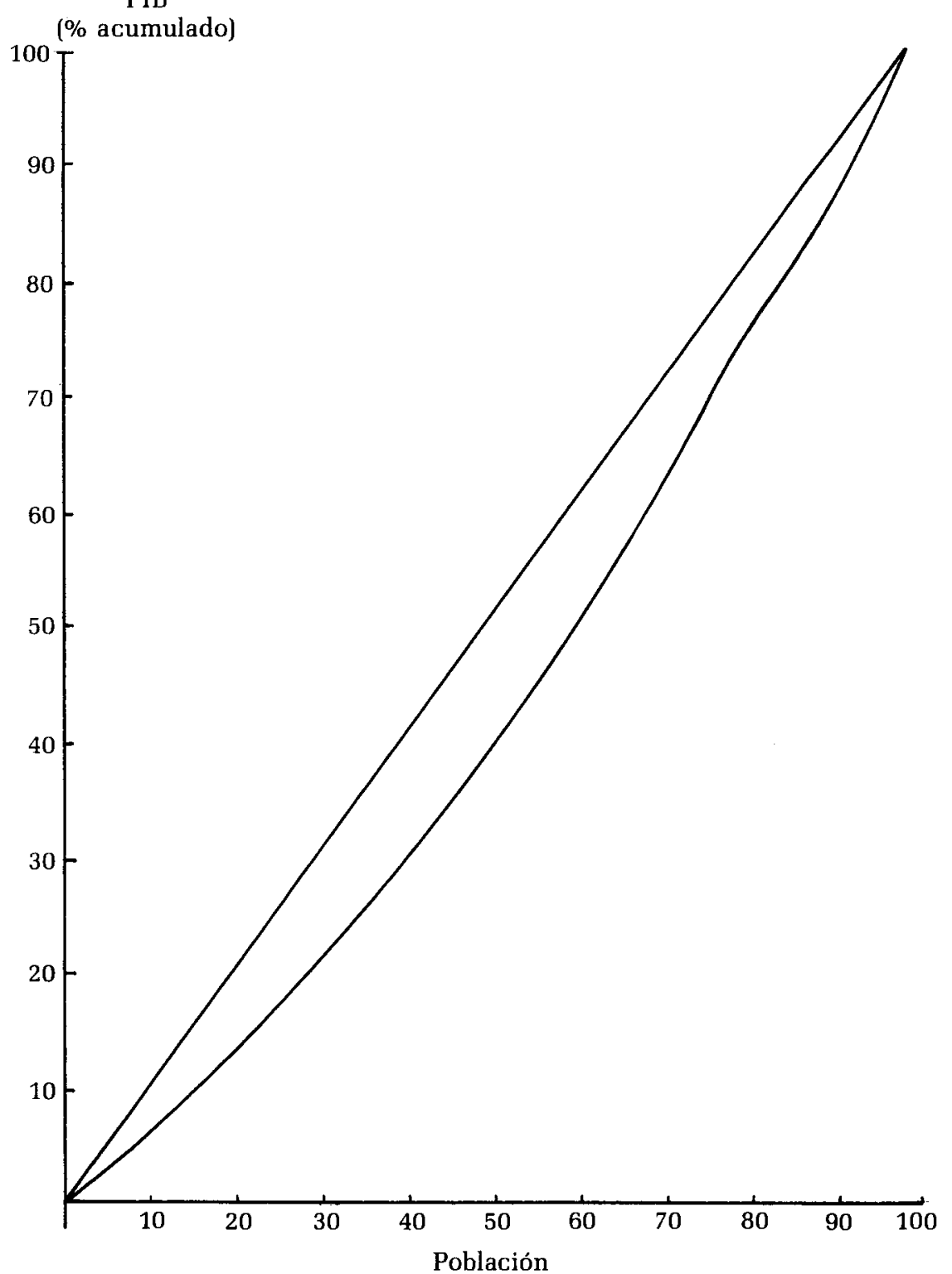

(\% acumulado) 
greso que se aproximan a la diagonal y otros que se alejan de ella en relación con la curva de Lorenz con que se hace la comparación, y como no se ha hecho explícito supuesto alguno sobre la importancia relativa de cada estrato, es imposible efectuar comparaciones del grado de desigualdad entre distribuciones. En estos casos, es probable que se obtengan ordenamientos distintos a partir de los diferentes índices.

Una conclusión importante que se deriva de lo anterior es que no es posible, en general, comparar el grado de desigualdad sin introducir juicios de valor sobre la distribución. Las diversas medidas denominadas positivas, llevan implícita una valuación de lo que se considera una distribución deseable del ingreso. Así, el coeficiente de Gini, por ejemplo, confiere una ponderación mayor a las transferencias hechas a niveles de ingreso cercanos a la moda, por ejemplo, estratos medios, y menor en los extremos.

Dado lo anterior, Atkinson $(1970$, p. 244) propone que estos juicios de valor sean considerados en forma explícita. Para ello construye una medida que parte de una función de bienestar social, que depende de un parámetro E, el cual representa la importancia relativa que la sociedad le confiere a la desigualdad. El parámetro toma el valor de cero cuando existe total indiferencia social a la desigualdad, y en el otro extremo, tiende a infinito cuando el único interés de la comunidad es la posición relativa del estrato de menor ingreso. La función de utilidad propuesta por Atkinson es de la forma ${ }^{6}$

$$
U(y \hat{i})=\frac{Y_{i}^{1-E}}{1-E} ; E=1
$$

donde $E$ es el coeficiente de aversión a la desigualdad y $Y i$ el $p_{i B}$ per cápita/indicador de bienestar del i-ésimo estado. A partir de esta función, se calcula el denominado ingreso equivalente igualmente distribuido $I_{E}$, que se define como el ingreso que distribuido equitativamente genera el mismo nivel de bienestar que el ingreso actual $I_{A}$ con su presente distribución. El índice de Atkinson compara estos dos tipos de ingresos mediante:

$$
\mathrm{A}=1-\frac{I_{E}}{I_{A}}
$$

${ }^{6}$ La función original propuesta por Atkinson es:

$$
U\left(Y_{i}\right)=A+B \frac{Y_{i}^{1-E}}{1-E} ; \text { se supone } A=O \text { y } B=1
$$


donde la razón $\frac{I_{E}}{I_{A}}$ representa el porcentaje del ingreso actual con el que se obtendrían el mismo nivel de bienestar presente si hubiese igualdad. De esta manera, el índice A se interpreta como la proporción de ingreso ineficientemente distribuido a raíz de la inequidad.

En los apartados siguientes se aplica la metodología descrita en esta sección. Primero se analiza la dinámica de la desigualdad del PIB per cápita estatal y, posteriormente, la relativa a los indicadores de bienestar.

La medición de la desigualdad del bienestar social, se realiza con base en cada uno de los indicadores que integran el índice compuesto de nivel de vida. No se utiliza el índice mismo ya que es una medida ordinal, no susceptible de este tipo de comparaciones intertemporales. Los coeficientes de Atkinson estimados para estas variables se utilizan como medidas del cambio en el grado de equidad. Su empleo como indicadores de desperdicio es de difícil interpretación y sus valores dependientes de la escala de medición de las variables, las cuales se expresan en tasas porcentuales.

\section{La desigualdad del PIB per cápita estatal}

Los datos del PIB per cápita revelan la existencia de notables diferencias en los niveles de desarrollo económico de las entidades federativas. ${ }^{7}$ El cuadro 1 muestra los resultados de la estratificación de este indicador.

En 1980, por ejemplo, el intervalo de mayor desarrollo lo componen únicamente Tabasco, el Distrito Federal y Nuevo León, dado que poseen niveles de PiB per cápita significativamente más elevados que, incluso, los de sus seguidores más cercanos. ${ }^{8} \mathrm{El}$ estrato medio lo conforman, en su mayor parte, entidades fronterizas del norte, así como los estados de México y Jalisco y sus respectivas entidades colindantes de Querétaro y Colima, y Quintana Roo y Chiapas, cuyas posiciones se analizan a continuación. El resto de los estados quedan comprendidos en el estrato de bajo desarrollo.

El ordenamiento de los estados denota claros patrones espaciales. Entre las diez entidades de mayor desarrollo se encuentran los estados de la frontera norte, el Distrito Federal, México y Jalis-

\footnotetext{
7 Se utilizan las cifras de INEGI (1985, p. 11).

${ }^{8}$ Tabasco se excluyó del ejercicio original de estratificación.
} 
Producto Interno Bruto per cápita por entidad federativa y estrato de desarrollo, 1970 y 1980

\begin{tabular}{|c|c|c|c|}
\hline \multicolumn{2}{|l|}{1970} & \multicolumn{2}{|l|}{1980} \\
\hline $\begin{array}{l}\text { Estrato de desarrollo } \\
\text { y entidad federativa }\end{array}$ & $\begin{array}{r}\text { PIB per } \\
\text { cápita } \\
\text { (millones \$) } \\
\end{array}$ & $\begin{array}{l}\text { Estrato de desarrollo } \\
\text { y entidad federativa }\end{array}$ & $\begin{array}{r}\text { PIB per cápita } \\
\text { (millones } \$ \text { ) } \\
\left.1970^{1}\right) \\
\end{array}$ \\
\hline Nacional $^{2}$ & 9094 & Nacional $^{2}$ & 12564 \\
\hline Estrato alto & 16311 & Estrato alto & 24735 \\
\hline Distrito Federal & 17634 & Tabasco $^{3}$ & 31382 \\
\hline Nuevo León & 15181 & Distrito Federal & 23935 \\
\hline Baja California & 13262 & Nuevo León & 19717 \\
\hline Sonora & 12661 & Estrato Medio & 13555 \\
\hline Baja California Sur & 12607 & Baja California & 16070 \\
\hline Estrato medio & 8908 & Baja California Sur & 15843 \\
\hline Coahuila & 10948 & Quintana Roo & 14920 \\
\hline México & 9718 & Coahuila & 14345 \\
\hline Tamaulipas & 9599 & Sonora & 13578 \\
\hline Jalisco & 9502 & Tamaulipas & 12872 \\
\hline Chihuahua & 9279 & Jalisco & 12619 \\
\hline Quintana Roo & 8900 & México & 12115 \\
\hline Sinaloa & 8528 & Chihuahua & 11829 \\
\hline Colima & 7826 & Colima & 11408 \\
\hline Morelos & 7658 & Chiapas & 10931 \\
\hline Campeche & 7606 & Querétaro & 10741 \\
\hline Veracruz & 7418 & Estrato bajo & 8197 \\
\hline Aguascalientes & 7175 & Aguascalientes & 9873 \\
\hline Querétaro & 7153 & Morelos & 9579 \\
\hline Estrato bajo & 5243 & Campeche & 9505 \\
\hline Nayarit & 6916 & Sinaloa & 9475 \\
\hline Tabasco & 6617 & Veracruz & 9062 \\
\hline Durango & 6576 & Durango & 9054 \\
\hline Yucatán & 6543 & Yucatán & 8966 \\
\hline Guanajuato & 6516 & Nayarit & 8890 \\
\hline Puebla & 5680 & Hidalgo & 8210 \\
\hline San Luis Potosí & 5344 & Puebla & 8139 \\
\hline Hidalgo & 4918 & Guanajuato & 8130 \\
\hline Michoacán & 4809 & San Luis Potosí & 7301 \\
\hline Zacatecas & 4730 & Michoacán & 6942 \\
\hline Guerrero & 4723 & Tlaxcala & 6901 \\
\hline Chiapas & 4520 & Guerrero & 6642 \\
\hline Tlaxcala & 4165 & Zacatecas & 5899 \\
\hline Oaxaca & 3237 & Oaxaca & 4990 \\
\hline
\end{tabular}

Fuente: Datos de INEGI [1985, p. 11] y estratificación propia.

${ }^{1}$ Se utilizó el deflactor implícito del PIB nacional del Sistema de Cuentas Nacionales de México.

${ }^{2}$ El PIB incluye aguas territoriales.

${ }^{3}$ El estado de Tabasco se excluyó del ejercicio de estratificación original. 
co. La región más rezagada comprende las entidades de la costa suroeste -Michoacán, Guerrero, Oaxaca y Chiapas- y los estados de Zacatecas y Tlaxcala.

Entre 1970 y 1980 la jerarquización de las entidades no varía en forma significativa. Cabe únicamente mencionar los casos de Tabasco, Chiapas y Quintana Roo como entidades de considerable ascenso en sus niveles de PiB per cápita. El acelerado crecimiento de los dos primeros estados se vincula con el impulso de la industria petrolera fomentado en dicha zona, mientras que el desarrollo de Quintana Roo se relaciona, sobre todo, con el dinamismo del turismo. El caso de Tabasco es particularmente notorio, ya que su PiB per cápita es dos y media veces superior a la media nacional en 1980 , encabezando la relación de entidades. Dado que la mayor parte de los ingresos petroleros no son apropiados por la entidad, sino que se distribuyen en el ámbito federal, con lo cual su producto está considerablemente sobreestimado y no es reflejo de su posición relativa de desarrollo, ${ }^{9}$ en los resultados que se presentan a continuación, se realiza el ejercicio de excluir a Tabasco del conjunto de estados para observar su incidencia sobre las conclusiones obtenidas de la dinámica de la desigualdad. La medición de las disparidades se realiza en función de tres aspectos: en términos de la totalidad de entidades, al interior de cada estrato de desarrollo entre conglomerados.

En el cuadro 2 se presentan los índices de desigualdad estimados para el PiB per cápita estatal. Los coeficientes de Gini calculados registran un valor de 0.22 en 1970 y 0.23 en 1980 . Esto parecería indicar que la distribución estatal del Producto Interno Bruto en el país permaneció sin cambio en la última década. Sin embargo, si se observa la gráfica 2 es posible apreciar que las curvas de Lorenz correspondientes a estos índices se intersectan, con lo cual esta conclusión no es válida. Cabe apuntar que si se excluye el estado de Tabasco de la estimación, se registra una disminución en el índice de Gini, si bien este resultado también queda sin validez.

Siguiendo la anterior argumentación, se calcularon índices de Atkinson del PiB estatal para diversos valores del coeficiente de aversión a la desigualdad E. Los resultados no registran variaciones significativas en el grado de equidad de la distribución del PIB por entidad federativa entre 1970 y 1980 . No obstante, al eliminar Tabasco se observa una disminución del grado de desigualdad durante la década para todos los valores de E considerados.

\footnotetext{
${ }^{9}$ Tabasco ocupa el lugar veintiocho entre las entidades federativas en lo relativo a su nivel de bienestar social.
} 
CUADRO 2

Índices de desigualdad del Producto Interno Bruto Estatal, 1970 y 1980

\begin{tabular}{|c|c|c|c|c|c|c|}
\hline \multirow[b]{2}{*}{ Año } & \multirow{2}{*}{$\begin{array}{c}\text { Coeficiente } \\
\text { de Gini }\end{array}$} & \multicolumn{5}{|c|}{ Índice de Atkinson (según vaior de $\mathrm{E}^{1}$ ) } \\
\hline & & 0.5 & 1.0 & 1.5 & 2.0 & 3.0 \\
\hline \multicolumn{7}{|c|}{$\begin{array}{l}\text { Total de } \\
\text { entidades } \\
\text { federativas }\end{array}$} \\
\hline 1970 & .2210 & .0383 & .0675 & .1090 & .1413 & .1994 \\
\hline 1980 & .2285 & .0426 & .0731 & .1136 & .1432 & .1942 \\
\hline \multicolumn{7}{|c|}{$\begin{array}{l}\text { Excluyendo } \\
\text { Tabasco }\end{array}$} \\
\hline 1970 & .2237 & .0391 & .0691 & .1120 & .1450 & .2046 \\
\hline 1980 & .1972 & .0308 & .0542 & .0871 & .1127 & .1593 \\
\hline
\end{tabular}

Fuente: Estimaciones propias con base en los datos del cuadro 1.

${ }^{1} \mathrm{E}$ es el coeficiente de aversión a la desigualdad.

Los índices de Atkinson estimados son también indicativos de los beneficios potenciales de una política redistributiva orientada a lograr total equidad en la distribución regional de producto en México. Así, por ejemplo, el índice de Atkinson para 1980, con $E=3.0$ y sin excluir Tabasco de la estimación, señala la posibilidad de obtener con una distribución perfecta del producto, el mismo nivel de bienestar social alcanzado en dicho año con el $\mathbf{8 0} \%$ del producto de 1980. Claro está que la magnitud del potencial redistributivo depende del grado de aversión a la desigualdad que se considere, con lo cual las conclusiones que se obtienen están en función del juicio de valor que se haga al respecto.

Continuando con el análisis de la desigualdad, los índices que se presentan en el cuadro 3 registran la evolución del grado de equidad al interior de los estratos definidos. Las entidades de mayor desarrollo relativo se vuelven más dispares durante la década, como lo denota, por ejemplo, el índice de Atkinson para $\mathrm{E}=1.5$, que pasa de 0.0125 en 1970 a 0.0269 en 1980 . Si se excluye Tabasco de la estimación, la desigualdad se reduce, al igual que en los casos anteriores. En cuanto a los otros intervalos, el grado de equidad del estrato medio permanece sin alteraciones y el correspondiente al bajo disminuye.

En materia de desigualdad entre grupos de entidades, la tasa de crecimiento media anual del PIB per cápita entre 1970 y 1980 
GRÁFICA 2

Curvas de Lorenz del producto interno bruto estatal 1970 y 1980 PIB

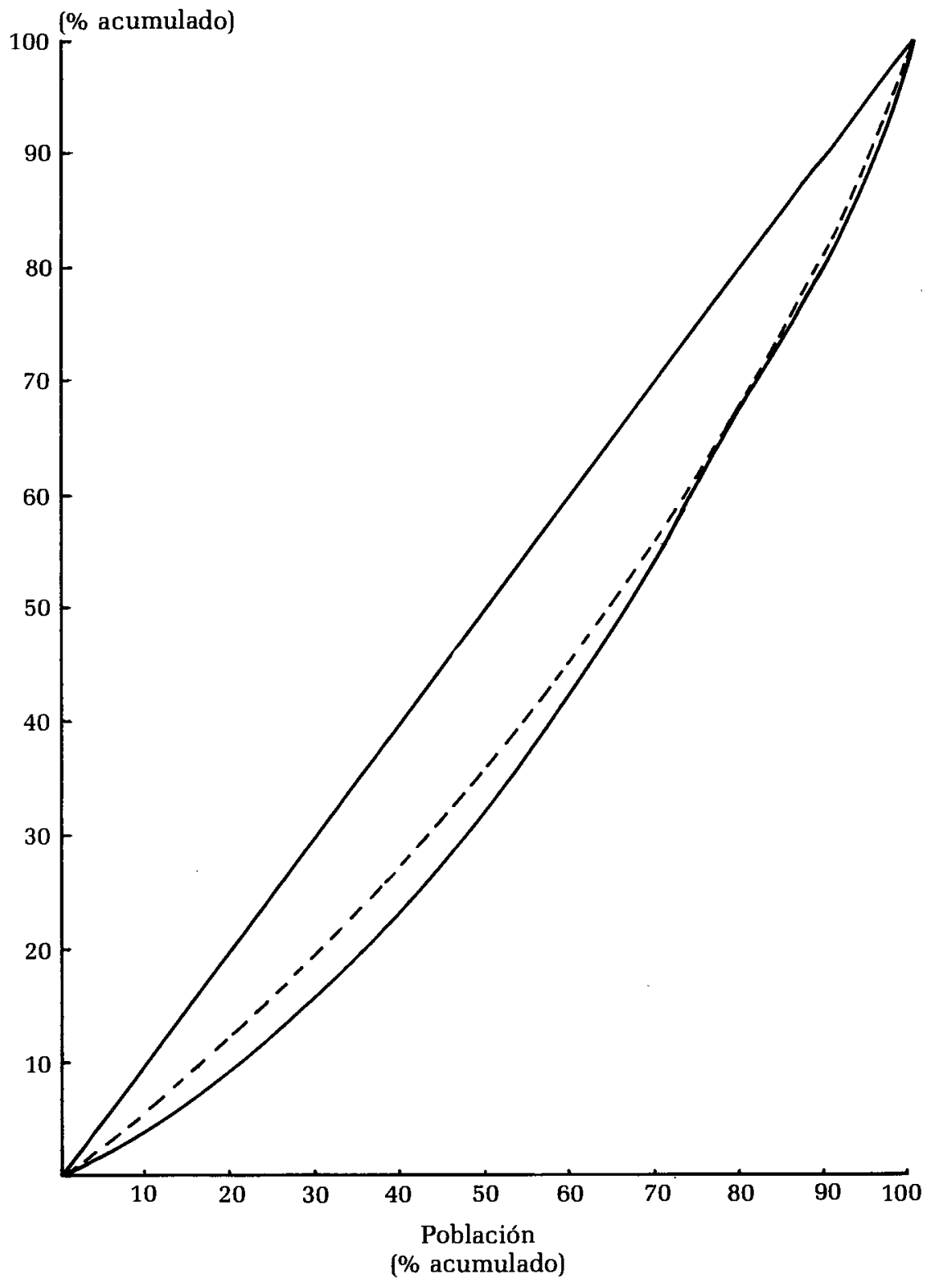


CUADRO 3

Índices de desigualdad del Producto Interno Bruto estatal por estrato de desarrollo, 1970 y 1980

\begin{tabular}{|c|c|c|c|c|c|c|}
\hline \multirow{2}{*}{$\begin{array}{l}\text { Estrato de } \\
\text { desarrollo y año }\end{array}$} & \multirow{2}{*}{$\begin{array}{c}\text { Coeficiente } \\
\text { de Gini }\end{array}$} & \multicolumn{5}{|c|}{ Índice de Atkinson (según valor de $\mathrm{E}^{1}$ ) } \\
\hline & & 0.5 & 1.0 & 1.5 & 2.0 & 3.0 \\
\hline \multicolumn{7}{|l|}{ Estrato alto } \\
\hline 1970 & .0705 & .0043 & .0077 & .0125 & .0164 & .0238 \\
\hline 1980 & .1036 & .0091 & .0163 & .0269 & .0355 & .0517 \\
\hline \multicolumn{7}{|l|}{ Estrato medio } \\
\hline 1970 & .0749 & .0044 & .0078 & .0130 & .0172 & .0253 \\
\hline 1980 & .0766 & .0044 & .0080 & .0132 & .0173 & .0258 \\
\hline \multicolumn{7}{|l|}{ Estrato bajo } \\
\hline 1970 & .1120 & .0104 & .0190 & .0323 & .0436 & .0669 \\
\hline 1980 & .0949 & .0078 & .0145 & .0250 & .0342 & .0537 \\
\hline
\end{tabular}

Fuente: Estimaciones propias con base en los datos del cuadro 1.

${ }^{1} \mathrm{E}$ es el coeficiente de aversión a la desigualdad.

proporciona una tendencia de lo ocurrido en este aspecto. Así, esta tasa alcanzó un valor de 4.3. Estos resultados sugieren una ligera reducción en la brecha prevaleciente entre las entidades de los niveles alto y medio de desarrollo y los estados de mayor pobreza relativa.

Disparidades estatales en los niveles de bienestar social

Dado que el fenómeno del desarrollo socioeconómico es fundamentalmente de carácter multivariado, en este apartado se dimensiona el nivel de bienestar social de las entidades federativas y la evolución de sus disparidades, con base en los siguientes once indicadores de ingresos, educación, vivienda y salud expresados en tasas porcentuales.

1. Población económicamente activa con ingresos iguales o superiores al salario mínimo.

2. Alfabetas de $\mathbf{1 0}$ años y más.

3. Población adulta con instrucción. ${ }^{10}$

4. Población adulta con primaria completa.

${ }^{10}$ Se refiere a la población de 15 años y más. 
5. Población adulta con enseñanza media.

6. Viviendas con piso diferente a tierra.

7. Viviendas con agua entubada.

8. Viviendas con tubería de drenaje.

9. Viviendas con energía eléctrica.

10. Viviendas con más de un cuarto.

11. Mortalidad. ${ }^{11}$

Los cuadros 4 y 5 presentan los indicadores de bienestar social estimados para cada entidad en 1970 y $1980 .{ }^{12}$ La información evidencia considerables desequilibrios regionales en los niveles de vida de la población del país. En materia de alfabetismo, por ejemplo, $92 \%$ en promedio de la población de 10 años y más de las entidades de mayor desarrollo sabía leer y escribir en 1980. En contraste, en Guerrero, Chiapas y Oaxaca 3 de cada 10 personas eran analfabetas. En relación con el nivel de ingresos, $81 \%$ de la fuerza de trabajo de los estados de alto bienestar percibió remuneraciones iguales o superiores al salario mínimo aproximado de 1980 , porcentaje mayor en $16 \%$ al de las entidades con niveles de vida medio y bajo. Finalmente, en cuanto a las condiciones de la vivienda, 8.4 de cada 10 contaron con agua entubada entre los estados del intervalo alto, en comparación con 5.4 de los de mayor pobreza. Es notorio el caso de Oaxaca, con apenas 4.6.

Con la finalidad de resumir en un número la información de bienestar social de cada entidad federativa, se construyó un índice compuesto de nivel de vida, aplicando el método de componentes principales a los datos de los cuadros 4 y $5 .{ }^{13}$ Estas últimas cifras sirvieron también de base para establecer tres agrupaciones de estados según su nivel de desarrollo relativo - alto, medio y bajo- utilizando la metodología de estratificación multivariada mencionada anteriormente. Los resultados aparecen en el cuadro 6 y se representan en el mapa 1 para el año de 1980.

Se observa, primeramente, que la estratificación es consistente con el ordenamiento del índice de componentes principales, ya que no existen traslapes entre el nivel relativo de los estados y la definición de los intervalos. La jerarquización de las entidades es, en general, similar a la obtenida con el PIB per cápita, con los es-

${ }^{11}$ Se utiliza 1000 menos la tasa bruta de mortalidad.

12 Las variables de 1970 se estimaron a partir de los datos de Dirección General de Estadística (1973) y de 1980 con base en iNEGi (1984).

${ }^{13}$ Se utilizaron los ponderadores de la primera componente, la cual explicó $58.2 \%$ de la variación total en 1970 y $60.8 \%$ en 1980 . Los ponderadores de cada variable se presentan en el anexo B. El índice se calculó aplicando estos pesos relativos a las variables originales normalizadas. 


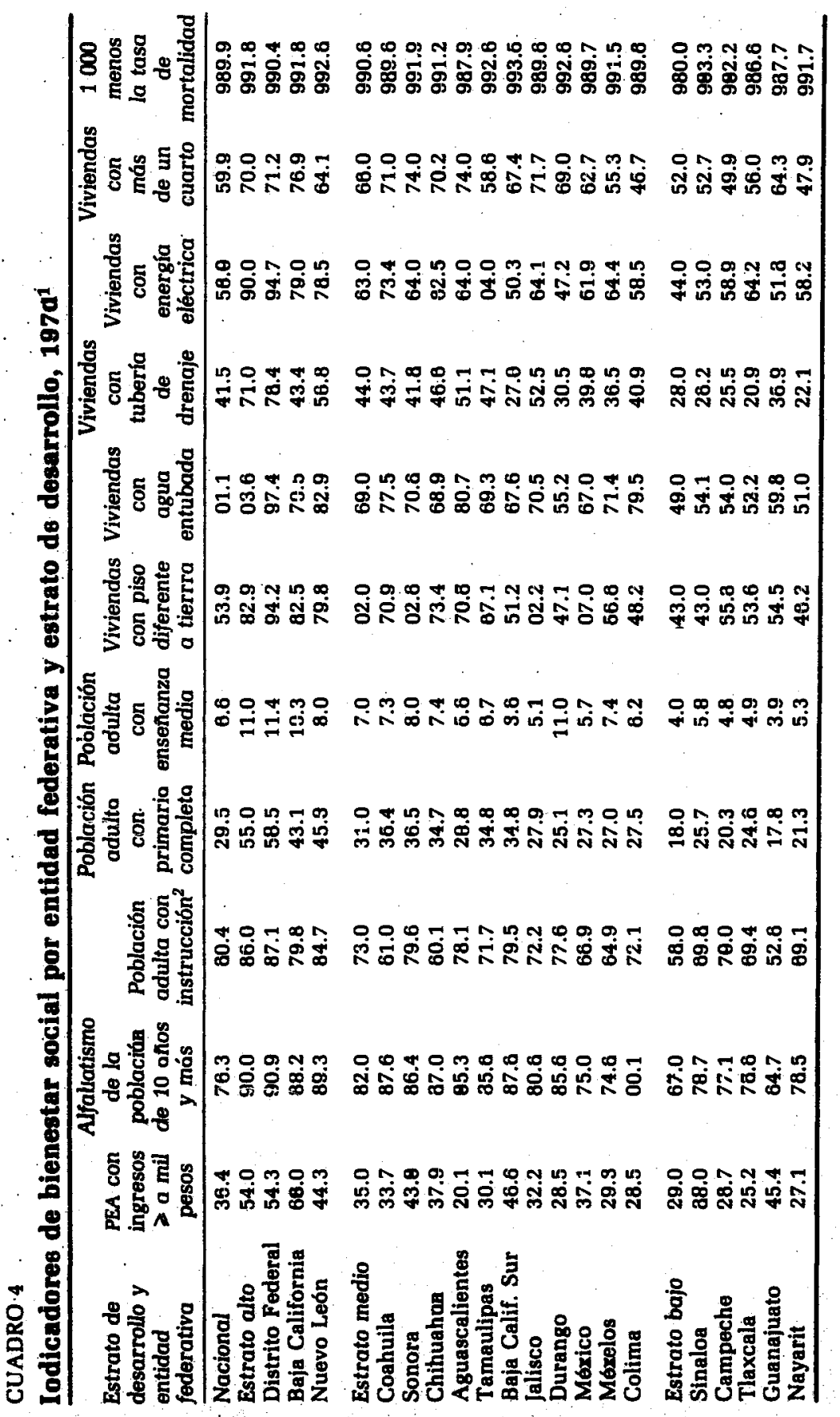




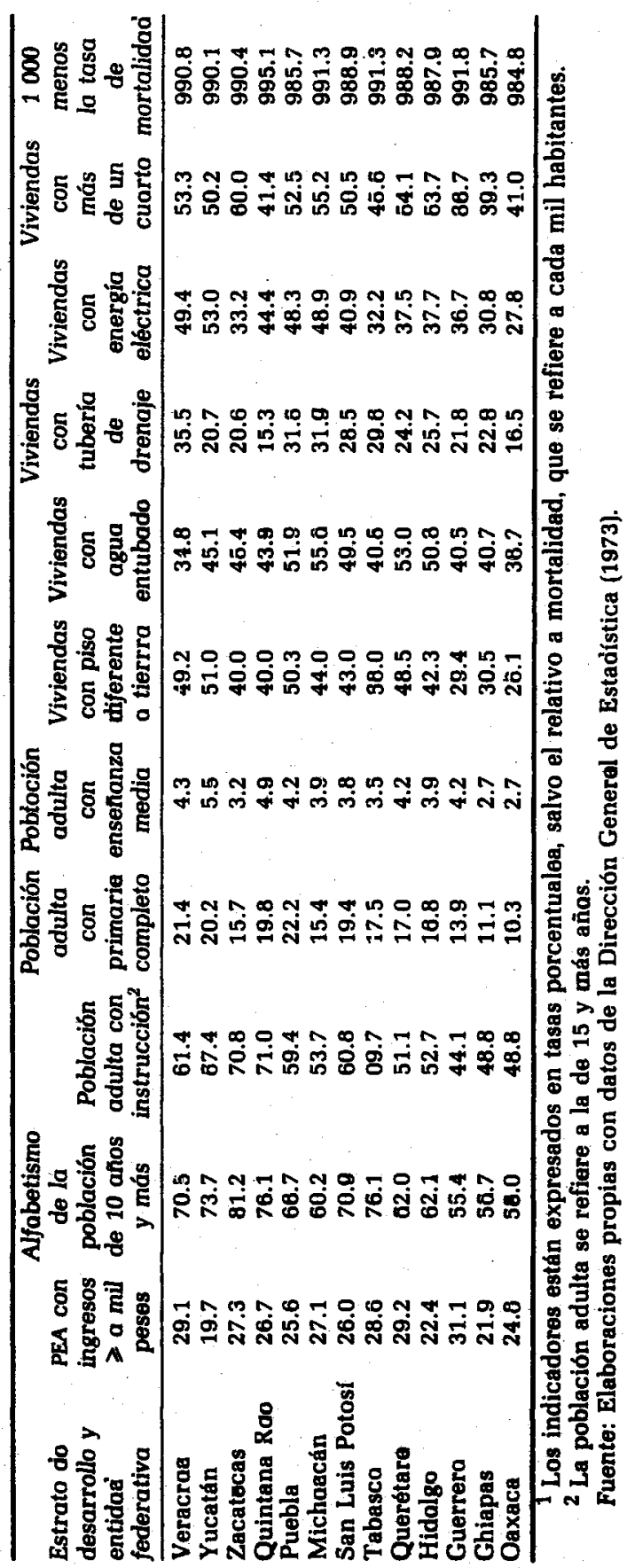




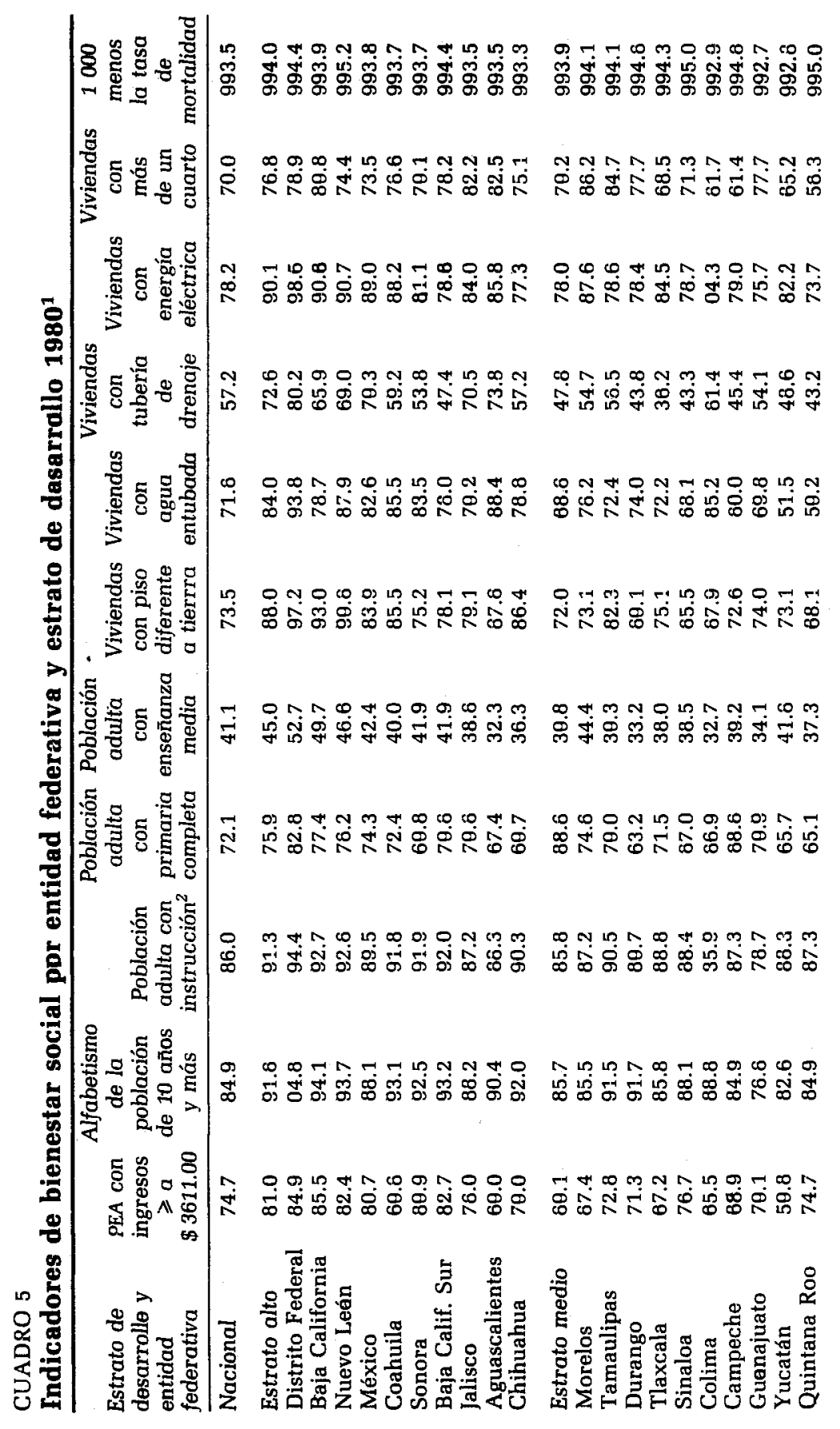




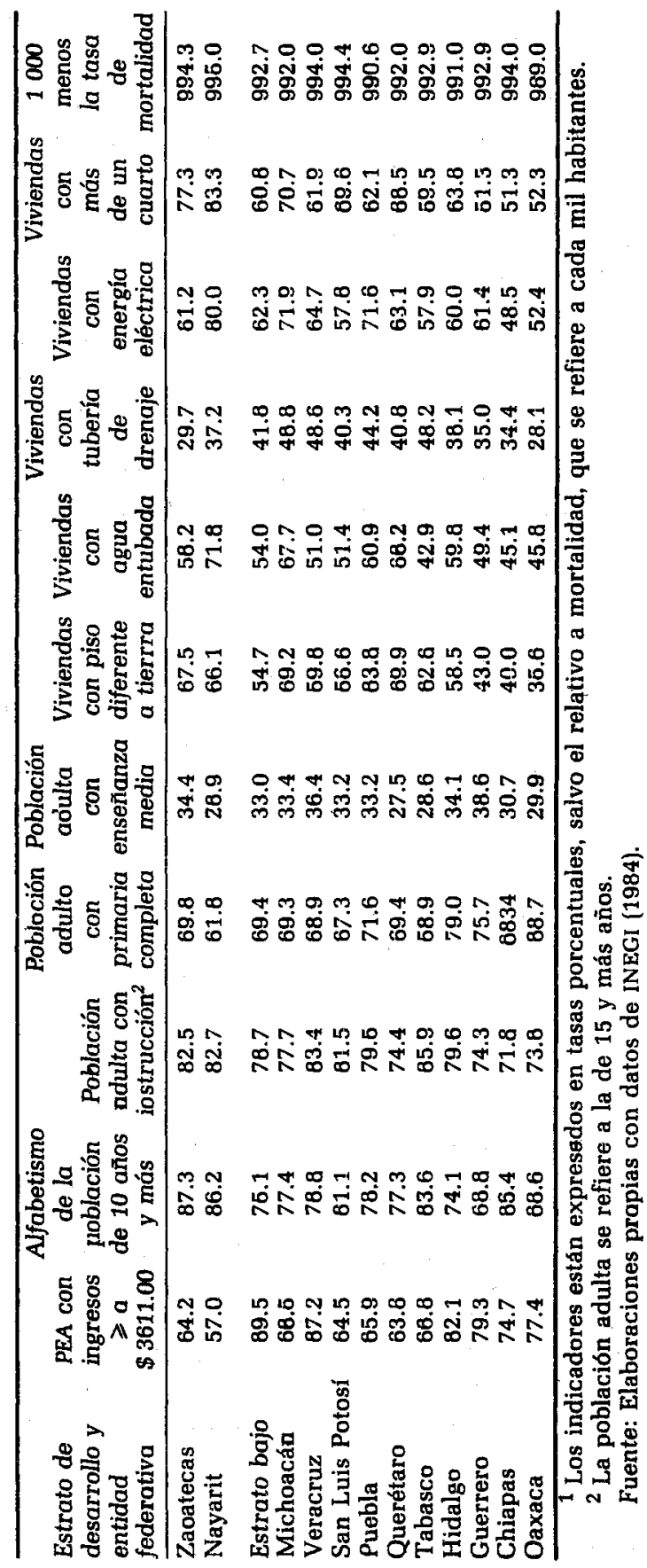


tados de la frontera norte, el Distrito Federal, México y Jalisco en los primeros sitios y las entidades de la costa suroeste entre los de mayor rezago. ${ }^{14}$ Cabe mencionar que, en el caso del índice de bienestar social, la estratificación de los estados, y no sólo su ordenamiento, denota claros patrones geográficos, en comparación con la del PiB per cápita.

Entre los casos de inconsistencia en las jerarquizaciones de los indicadores y del PiB per cápita, cabe señalar los siguientes. Sobresale Tabasco que, pese a haber registrado el PiB per cápita más alto de las entidades federativas en 1980, es el quinto estado de mayor pobreza relativa en términos de las condiciones del nivel de vida de su población. Son notables también los casos de Quintana Roo, con el sexto sitio en nivel de PiB per cápita y el vigésimo en bienestar social; Querétaro con los lugares quinceavo y vigesimoséptimo, respectivamente; Chiapas con 140. y 310.; México 11o. y 40.; Aguascalientes, 16o. y 9o. y Morelos, 17o. y 110.

Comparando las posiciones relativas de nivel de vida a lo largo de los setenta, destaca el progreso del estado de México, que pasa del doceavo sitio en 1970 a ubicarse, al término de la década, entre las cuatro entidades de mayor desarrollo. Sobresalen también entre los estados en ascenso, Tlaxcala y Quintana Roo; mientras que Chihuahua, Tamaulipas, Veracruz y Nayarit registraron caídas en sus posiciones relativas de bienestar.

Los resultados de la estratificación revelan un sensible aumento en el número de entidades comprendidas en el intervalo de alto desarrollo, pasando de apenas 3 en 1970 a 10 en 1980, así como una considerable reducción del grupo de estados de mayor pobreza, de 18 a 10 entidades. Esto sugiere una más amplia distribución espacial de los beneficios del desarrollo, con un mayor número de entidades de alto bienestar y menos estados de bajo nivel de vida.

El cuadro 7 contiene los índices de desigualdad estimados para cada uno de los once indicadores del bienestar social considerados. Los resultados se caracterizan por una notable consistencia. Los índices de Gini y Atkinson, calculados para las once variables apuntan, en todos los casos, hacia una disminución significativa del grado de desigualdad en los niveles de vida de las entidades federativas entre 1970 y 1980. A manera de ejemplo, para el indicador de población adulta con primaria completa, el índice de Gini se reduce de 0.221 en 1970 a 0.033 en 1980 y el de

14 La correlación gloBal entre el índice de bienestar social y el PIB per cápita es de 0.895 en 1970 y 0.738 en $\mathbf{1 9 8 0}$. Este último ano sin incluir a TaBasco. 
CUADRO 6

Índice de bienestar social por entidad federativa y estrato de desarrollo, 1970 y 1980

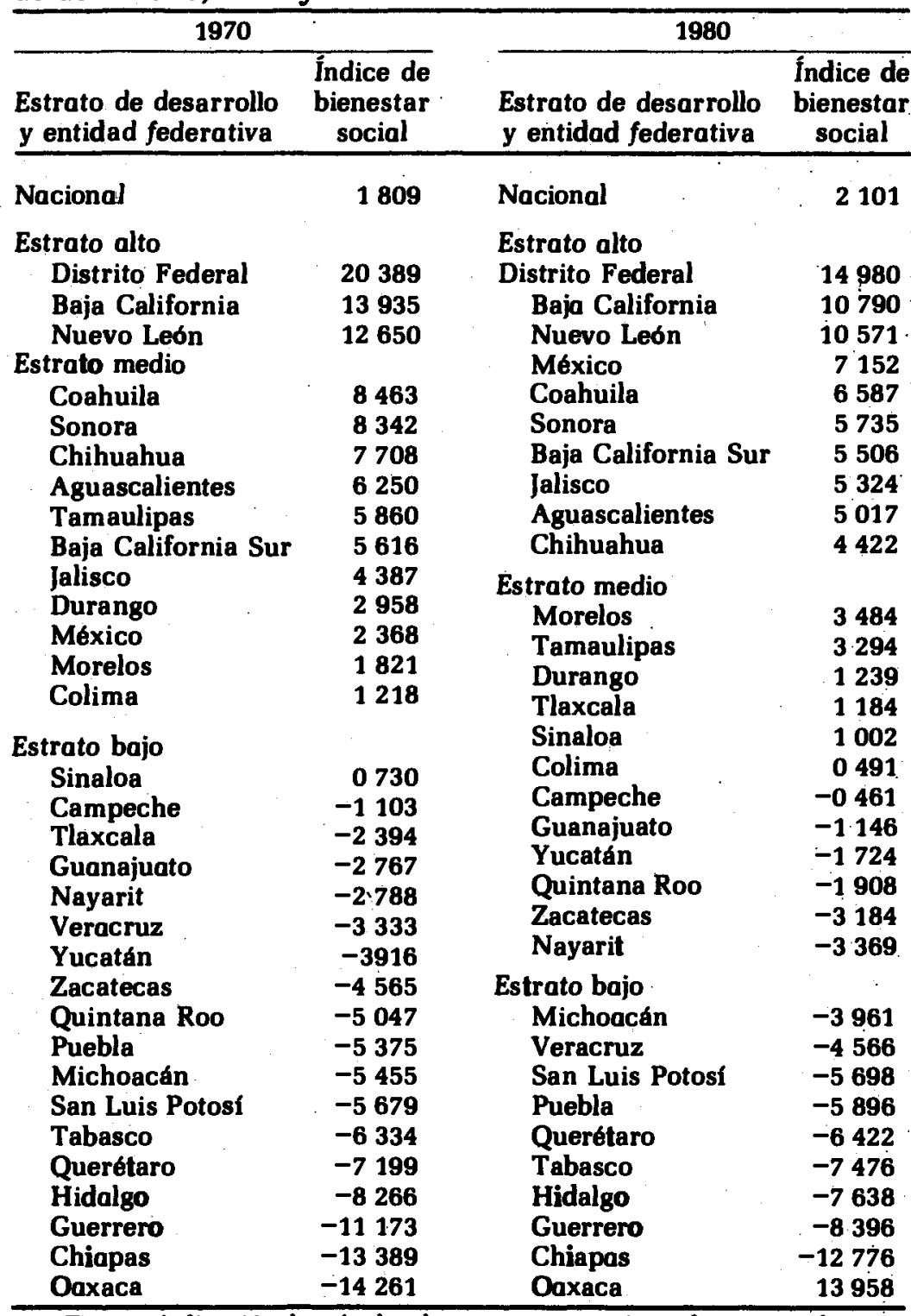

Fuente: Aplicación de métodos de componentes principales y estratificación multivariada a los datos de los cuadros 4 y 5. 
MAPA 1

Zonas de desarrollo según niveles de bienestar social de las entidades federativas 1980

Nivel de Bienestar Social

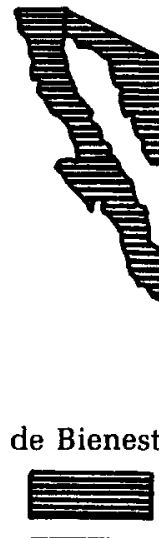

Medio

$\because \because \because \cdots$

Bajo

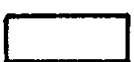

Atkinson pasa de 0.384 a 0.0010 , con $\mathrm{E}=0.5$, y de 0.2094 a 0.0062 con $\mathrm{E}=3.0$.

Los resultados por estrato se aprecian en los cuadros 8 y 9. Las entidades comprendidas en el intervalo de alto bienestar registran una disminución del grado de desigualdad en la mayor parte de los indicadores. Únicamente en las variables de población adulta con enseñanza media y de viviendas con piso diferente a tierra el grado de inequidad no varía, y en el caso de alfabetismo se observa un incremento. En los estratos medio y bajo se presenta también una significativa reducción de la desigualdad al interior de cada grupo para 9 de los 11 indicadores. Sólo en el caso de viviendas con agua entubada, la inequidad aumenta en ambos estratos y en los indicadores de viviendas con tuberías de drenaje y con piso diferente a tierra no se registran variaciones para los intervalos medio y bajo, respectivamente.

Finalmente, el cuadro 10 incluye las tasas de crecimiento promedio anual por estrato para cada una de las variables de bienestar estudiadas. Estas estimaciones se utilizan como indicativas de la evolución del grado de desigualdad entre grupos de entidades. En primer término, destaca el hecho de que la totalidad de tasas 
de crecimiento asumen valores positivos, lo cual pone en evidencia el proceso de desarrollo del país durante la década de los años setenta, que se tradujo en una mejoría general de los niveles de vida de la población.

Los ritmos más elevados de crecimiento se registran entre las entidades del estrato de mayor pobreza relativa, seguidos de los intervalos medio y alto. De esta manera, los avances en materia de bienestar social fueron proporcionalmente mayores en las entidades de menor nivel de vida, lo cual indica una relativa disminución de la brecha entre estados pobres y de alto bienestar. A manera de ejemplo, el porcentaje de adultos con primaria completa en las entidades de menor desarrollo creció a un ritmo anual de $15 \%$ en la década. En cambio, en los estados de mayor nivel de vida el incremento fue de apenas $3.3 \%$. Las entidades del estrato medio se ubicaron entre estos dos valores, con un aumento de $8.3 \%$. Situaciones similares se observan en el resto de los indicadores.

\section{Conclusiones}

La aplicación de metodología de estratificación multivariada permitió la obtención de agrupaciones de entidades federativas con características homogéneas en sus niveles de desarrollo, definidos en términos del producto interno bruto per cápita y de once indicadores de bienestar social para 1970 y 1980 . En el caso de estos últimos se construyó un índice compuesto de nivel de vida con la técnica de componentes principales, con el objeto de ordenar a las entidades según sus niveles relativos de bienestar.

Los resultados de la jerarquización de estados son, en general, similares en términos de ambas definiciones del desarrollo. A lo largo de la década de los setenta, encabezan la relación de entidades los estados fronterizos del norte, el Distrito Federal, México y Jalisco. En el otro extremo, las entidades con menores niveles de PIB per cápita y bienestar social se concentran en la región de la costa sur del Pacífico: Guerrero, Oaxaca y Chiapas.

Cabe mencionar el caso de Tabasco como una entidad de niveles particularmente contrastantes de PIB per cápita y bienestar social, ya que ocupa la primera posición en términos del primer indicador en 1980 y, en cambio, la número veintiocho en sus condiciones de nivel de vida. La inconsistencia obedece, en buena medida, a la contabilización de los ingresos petroleros en el PIB de la entidad, cuando que éstos se distribuyen, en su mayor parte, en el ámbito federal.

Los índices estimados de Gini y Atkinson para el PIB per cápi- 
CUADRO 7

Indices de desigualdad de los indicadores de bienestar social de las entidades federativas, 1970 y 1980

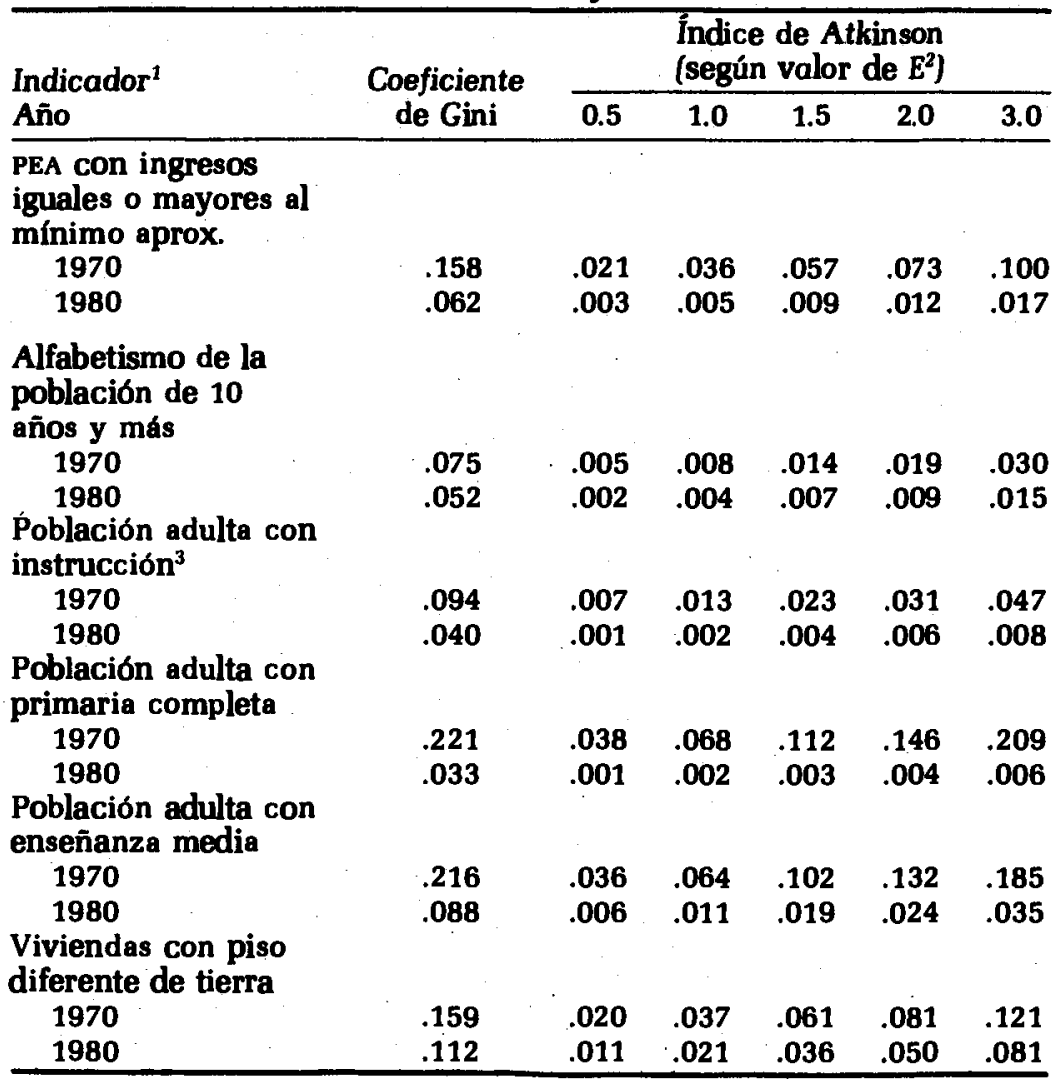

ta estatal -excluyendo Tabasco- y cada uno de los indicadores de bienestar social considerados, registran una disminución del grado de desigualdad en la distribución espacial del desarrollo durante los años setenta, tanto en términos globales, como al interior de cada estrato y entre los grupos de entidades definidos. ${ }^{15}$ Los resultados de otros estudios históricos del desarrollo regional del país, apuntan hacia una tendencia divergente de las disparidades

15 Si se considera el estado de Tabasco en las estimaciones relativas al PIB per cápita, el grado de desigualdad estatal no registra variaclones entre 1970 y 1980. 
CUADRO 7 (continuación)

\begin{tabular}{|c|c|c|c|c|c|c|}
\hline \multirow{2}{*}{$\begin{array}{l}\text { Indicador }{ }^{1} \\
\text { Año }\end{array}$} & \multirow{2}{*}{$\begin{array}{l}\text { Coeficiente } \\
\text { de Gini }\end{array}$} & \multicolumn{5}{|c|}{$\begin{array}{l}\text { Índice de Atkinson } \\
\text { (según valor de } E^{2} \text { ) }\end{array}$} \\
\hline & & 0.5 & 1.0 & 1.5 & 2.0 & 3.0 \\
\hline \multicolumn{7}{|c|}{$\begin{array}{l}\text { Viviendas con agua } \\
\text { entubada }\end{array}$} \\
\hline 1970 & .136 & .014 & .026 & .043 & .056 & .082 \\
\hline 1980 & .117 & .011 & .020 & .034 & .045 & .069 \\
\hline \multicolumn{7}{|c|}{$\begin{array}{l}\text { Viviendas con tub. } \\
\text { de drenaje }\end{array}$} \\
\hline 1970 & .207 & .034 & .060 & .096 & .125 & .176 \\
\hline 1980 & .152 & .018 & .032 & .053 & .069 & .101 \\
\hline \multicolumn{7}{|c|}{$\begin{array}{l}\text { Viviendas con } \\
\text { energía eléctrica }\end{array}$} \\
\hline 1970 & .158 & .020 & .036 & .061 & .082 & .122 \\
\hline 1980 & .091 & .007 & .013 & .022 & .029 & .046 \\
\hline \multicolumn{7}{|c|}{$\begin{array}{l}\text { Viviendas con más } \\
\text { de un cuarto }\end{array}$} \\
\hline 1970 & .109 & .009 & .017 & .028 & .038 & .057 \\
\hline 1980 & .074 & .004 & .008 & .013 & .018 & .028 \\
\hline \multicolumn{7}{|c|}{$\begin{array}{l}1000 \text { menos la tasa } \\
\text { Bruta de mortalidad }\end{array}$} \\
\hline $1970^{4}$ & 1.366 & .020 & .004 & .005 & .006 & .009 \\
\hline $1980^{4}$ & .627 & .010 & .010 & .002 & .002 & .002 \\
\hline
\end{tabular}

Fuente: Elaboraciones propias con los datos de los cuadros 4 y 5

1 Los indicadores se consideraron expresados en tasas porcentuales.

$2 \mathrm{E}$ = coeficiente de aversión a la desigualdad.

3 La población adulta se refiere a la de 15 y más años.

${ }^{4}$ Los indices de Atkinson son por $10^{-3}$

regionales entre 1940 y $1970 .{ }^{16}$ Ello hace pensar que la convergencia iniciada en la última década es un fenómeno reciente, cuya permanencia es aún difícil de prever.

En todo caso, es necesario continuar revirtiendo las tendencias concentradoras derivadas de la etapa del desarrollo nacional que se inicia en la década de los cuarenta. En el plano espacial, los beneficios potenciales de una política redistributiva del producto en México parecen ser bastante claros. En 1980, por ejemplo, los índices de desigualdad estimados señalan la posibilidad

16 Entre los estudios que analizan la evolución histórica de la desigualdad regional en México, se encuentran Unikel y Victoria (1970), Scott (1982) y Hernández Laos (1984). 
CUADRO 8

Índices de Gini de los indicadores de bienestar social por estrato de desarrollo, 1970 y 1980

\begin{tabular}{|c|c|c|c|}
\hline \multirow[b]{2}{*}{ Indicador $^{1}$} & \multicolumn{3}{|c|}{ Estrato de desarrollo } \\
\hline & Alto & Medio & Bajo \\
\hline \multicolumn{4}{|c|}{$\begin{array}{l}\text { PEA con ingresos iguales } \\
\text { o mayores al salario } \\
\text { mínimo aproximado }\end{array}$} \\
\hline 1970 & .0970 & .0967 & .1008 \\
\hline 1980 & .0487 & .0462 & .0448 \\
\hline \multicolumn{4}{|c|}{$\begin{array}{l}\text { Alfabetismo de la población } \\
\text { de } 10 \text { años y más }\end{array}$} \\
\hline 1970 & .0067 & .0294 & .0658 \\
\hline 1980 & .0133 & .0219 & .0416 \\
\hline \multicolumn{4}{|c|}{$\begin{array}{l}\text { Población adulta con } \\
\text { instrucción }{ }^{2}\end{array}$} \\
\hline 1970 & .0193 & .0392 & .0836 \\
\hline 1980 & .0128 & .0203 & .0318 \\
\hline \multicolumn{4}{|c|}{$\begin{array}{l}\text { Población adulta con } \\
\text { primaria completa }\end{array}$} \\
\hline 1970 & .0696 & .0769 & .1404 \\
\hline 1980 & .0322 & .0294 & .0279 \\
\hline \multicolumn{4}{|c|}{$\begin{array}{l}\text { Población adulta con } \\
\text { enseñanza media }\end{array}$} \\
\hline 1970 & .0763 & .1217 & .1148 \\
\hline 1980 & .0768 & .0626 & .0564 \\
\hline \multicolumn{4}{|c|}{$\begin{array}{l}\text { Viviendas con piso } \\
\text { diferente de tierra }\end{array}$} \\
\hline 1970 & .0374 & .0823 & .1046 \\
\hline 1980 & .0434 & .0349 & .1048 \\
\hline
\end{tabular}

de haber obtenido el mismo nivel de bienestar alcanzado en dicho año con el $\mathbf{8 0} \%$ del producto generado, si se hubiesen distribuido en forma equitativa los beneficios de la producción.

\section{Anexo estadístico}

A. Método de estratificación multivariada ${ }^{17}$

El método de estratificación multivariada utilizado es una técnica que permite formar agrupaciones de individuos en función al gra-

\footnotetext{
${ }^{17}$ Рara una descripción formal de este método, véase Jarque (1981, 163-169).
} 
CUADRO 8 (continuación)

\begin{tabular}{|c|c|c|c|}
\hline \multirow{2}{*}{ Indicador ${ }^{1}$} & \multicolumn{3}{|c|}{ Estrato de desarrollo } \\
\hline & Alto & Medio & Bajo \\
\hline \multicolumn{4}{|c|}{$\begin{array}{l}\text { Viviendas con agua } \\
\text { entubada }\end{array}$} \\
\hline 1970 & .0715 & .0499 & .0720 \\
\hline 1980 & .0329 & .0742 & .0884 \\
\hline \multicolumn{4}{|c|}{$\begin{array}{l}\text { Viviendas con tubería } \\
\text { de drenaje }\end{array}$} \\
\hline 1970 & (1306 & .1026 & .1257 \\
\hline 1980 & .0906 & .1092 & .0927 \\
\hline \multicolumn{4}{|c|}{$\begin{array}{l}\text { Viviendas con energía } \\
\text { eléctrica }\end{array}$} \\
\hline 1970 & .0428 & .0574 & .1328 \\
\hline 1980 & .0395 & .0416 & .0653 \\
\hline \multicolumn{4}{|c|}{$\begin{array}{l}\text { Viviendas con más de } \\
\text { un cuarto }\end{array}$} \\
\hline 1970 & .0402 & .0695 & .0840 \\
\hline 1980 & .0222 & .0558 & .0647 \\
\hline \multicolumn{4}{|c|}{$\begin{array}{l}1000 \text { menos la tasa bruta } \\
\text { de mortalidad }\end{array}$} \\
\hline 1970 & .0005 & .0009 & .0016 \\
\hline 1980 & .0003 & .0005 & .0008 \\
\hline
\end{tabular}

Fuente: Elaboraciones propias con los datos de los cuadros 4 y 5.

1 Los indicadores se consideraron expresados en tasas porcentuales.

2 La población adulta se refiere a la de 15 años y más.

do de homogeneidad que guarden con respecto a un conjunto de atributos comunes. En el caso del presente trabajo, el objetivo es construir conglomerados de entidades federativas con condiciones similares en sus niveles de bienestar social.

El método parte de una matriz de $n$ renglones (entidades) por $k$ columnas (indicadores de bienestar), donde $X_{i j}$ denota la i-ésima observación de la j-ésima variable. ${ }^{18}$ En primer término, se ordenan las observaciones de cada columna de menor a mayor valor y se transforman a un rango de cero a cien, mediante $Y_{i j}=a+$ $\mathrm{b} X_{i j}$; donde:

$$
\mathrm{b}=\frac{100}{\operatorname{máx}_{i}\left(X_{i j}\right)-\min _{i}\left(X_{i j}\right)} a=-(b) \min \left(X_{i j}\right)
$$

${ }^{18}$ Los datos respectivos para el caso de este trabajo son los presentados en los cuadros 4 y 5 . 
CUADRO 10

Tasas de crecimiento 1970-1980 de los indicadores de bienestar social por estrato de desarrollo

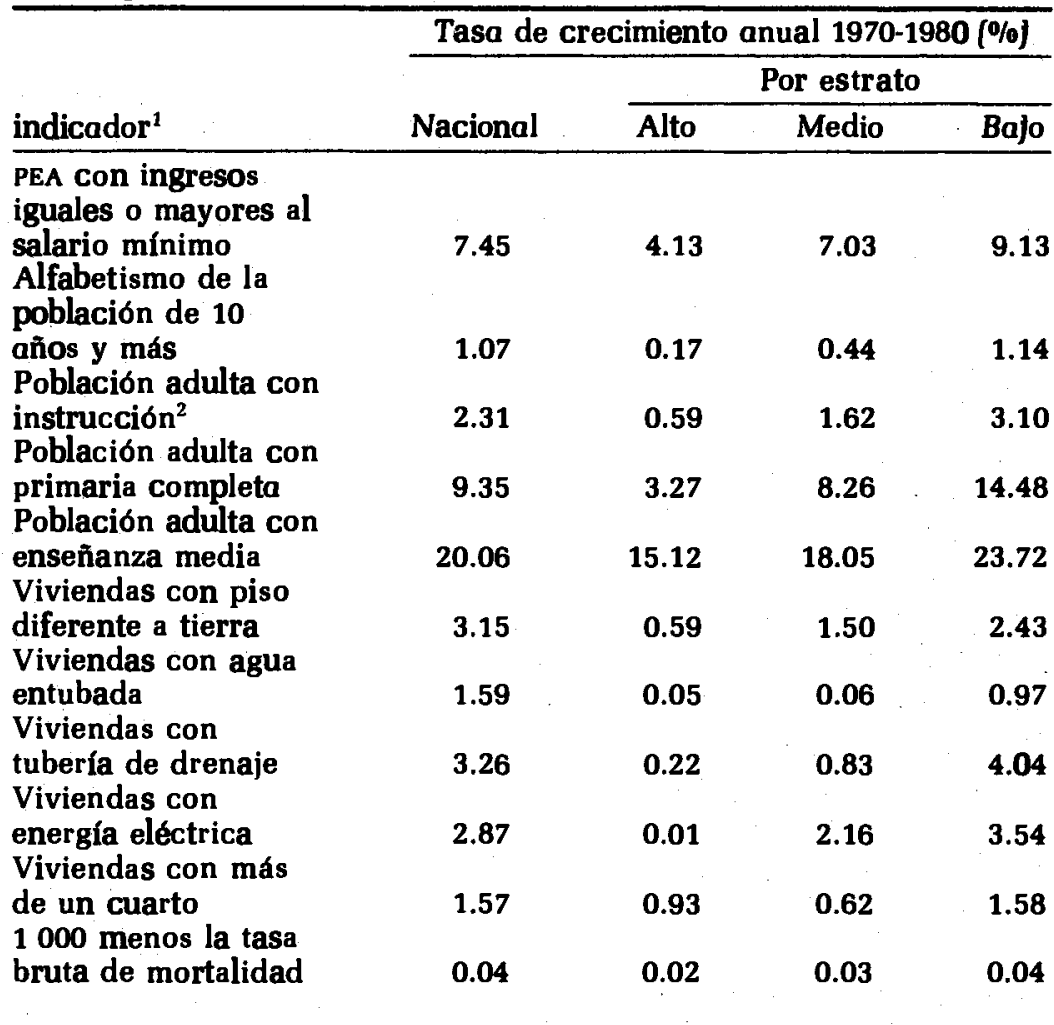

Fuente: Elaboraciones propias con los datos de los cuadros 4 y 5.

${ }^{1}$ Los indicadores se consideraron expresados en tasas porcentuales.

2 La población adulta se refiere a la de 15 y más años.

A los datos así transformados, se les aplica el método de estratificación univariada de Dalenuis, que posibilita la construcción de conglomerados de varianza mínima para cada indicador.

El procedimiento en que se fundamenta esta técnica, parte de la formación de diez intervalos de igual longitud con los valores en rango 0-100. Con base en esta agrupación, se calculan las raices cúbicas de las frecuencias absolutas de cada estrato y se obtienen sus totales acumulados. Con esta información, se estiman los 
límites óptimos de los conglomerados que sé desea formar, mediante:

$$
X^{(L-1)}=\frac{(L-1) T}{L}
$$

donde $T$ es el total acumulado de las raíces cúbicas de las frecuencias, $L$ el número de estratos y $X^{(L-1)}$ es el límite del estrato $L-1$.

El método de Dalenius da por resultado una estratificación de límites óptimos para el caso uniparamétrico. El problema de formación de conglomerados a partir de múltiples variables, consiste en definir $L$ estratos que satisfagan cierto criterio de optimalidad. Para ello, se supone que $O_{1}, \ldots, O_{k}$ son los $K$ parámetros a estimar, que corresponden a las medias poblacionales de las $k$ variables consideradas. El objetivo, entonces, es contruir los $L$ estratos que, dado un tamaño de muestra fija n, minimicen la función de las varianzas de los estimadores dada por:

$$
G(c)=\sum_{k} e_{k, c}=\sum_{k} \frac{v_{c}\left(\hat{O}_{k}\right)}{V^{*}\left(\hat{O}_{k}\right)} k=1, \ldots, k
$$

donde: $V_{c}\left(\hat{O}_{k}\right)$ es la varianza que resulta mediante una estratificación dada $C$, y $V^{*}\left(\hat{O}_{k}\right)$ es la varianza obtenida a través de la aplicación del procedimiento de Dalenius a cada variable. ${ }^{19}$ En general, los valores $V^{*}\left(O_{1}\right), \ldots, V^{*}\left(O_{k}\right)$, denominados varianzas mínimas, son inferiores a las varianzas obtenidas mediante el uso de cualquier otra estratificación, por lo que los cocientes $\mathrm{e}_{\mathrm{k}, c}$ se pueden considerar "eficiencias" de la estratificación $C$ con respecto a los $k$ estimadores.

Para la minimización de G(c), se utiliza un algoritmo computacional que requiere como información inicial primero la matriz de observaciones originales, transformadas según $Z_{i, k}=\frac{X_{i k}}{\left[V^{*}\left(\hat{O}_{k}\right)\right]^{1 / 2}}$, estandarización que se deriva de la optimización de $G(c)$; después, el número de conglomerados que se desea formar y, por último, los centros iniciales de los estratos, que por conveniencia, son las primeras L observaciones. Primeramente, se asigna cada observación al estrato cuyo centro es el más cercano, calculando para ello

19 Para el cálculo de las varianzas, se supone que se obtiene una muestra de tamaño n de una población de $N$ elementos y que los parámetros a estimar son las k medias poblacionales $O, \ldots, O_{k}$ de un cierto conjunto de variables. El estimador de $O$ utiliza muestreo aleatorio estratificado y la varianza * de $O$ emplea asignación proporcional del tamaño de muestra y considera el factor de corrección para poblaciones finitas. 


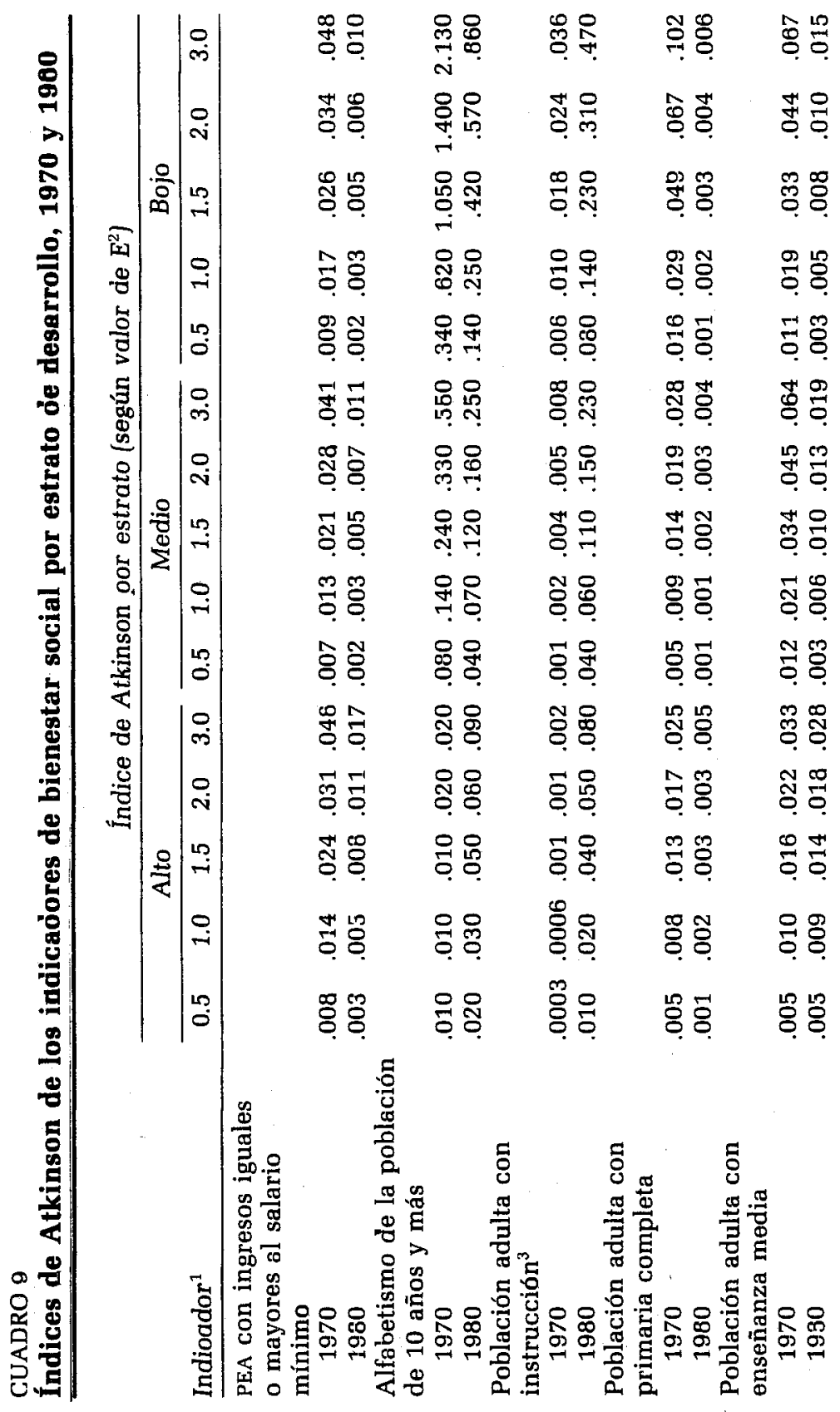




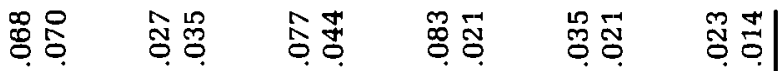

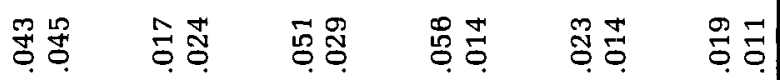

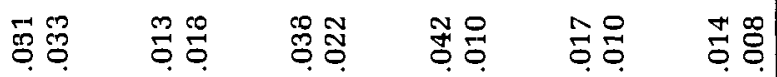

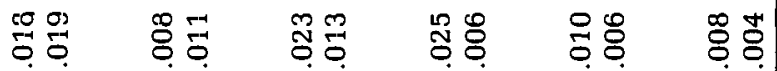

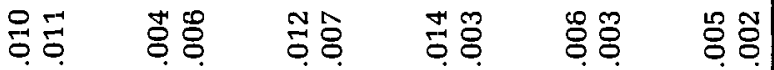

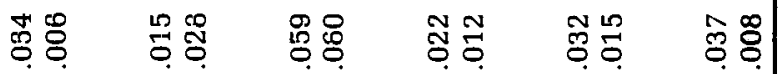

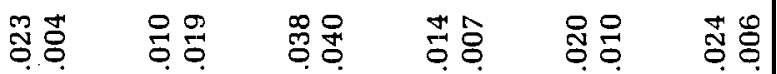

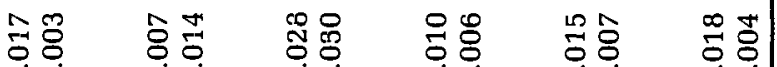

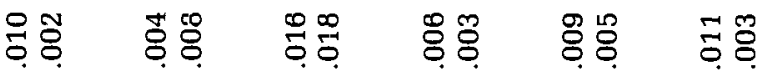

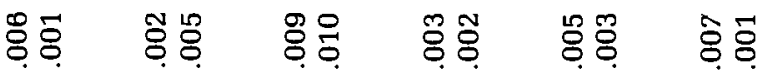

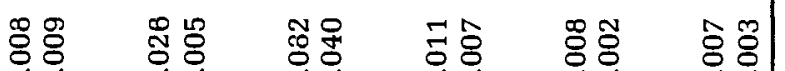

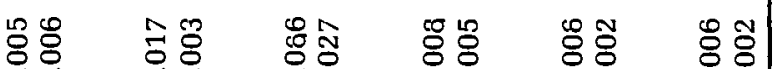
ఫัँ

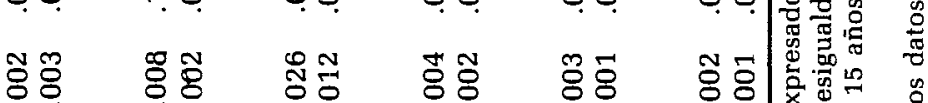

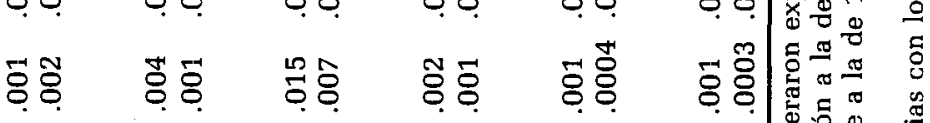

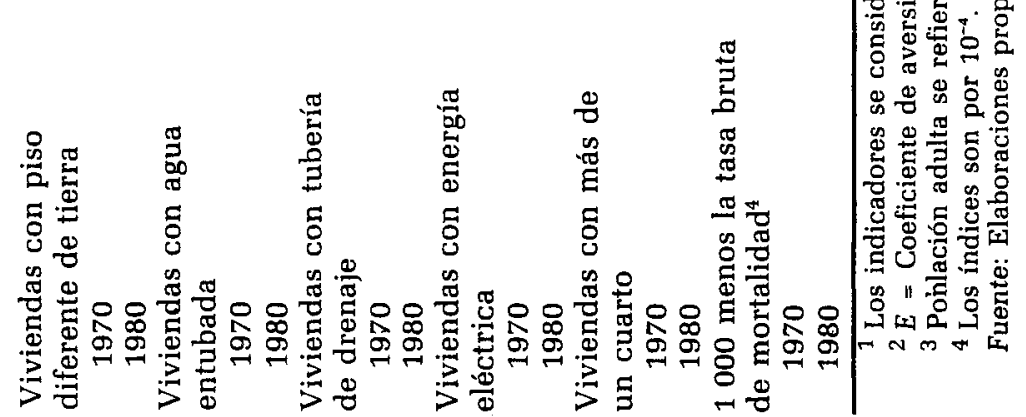


la distancia entre la i-ésima variable y los $L$ centros iniciales mediante:

$$
D_{M}=\sum_{k}\left(Z_{i, k}-C_{m, k}\right)^{2} \quad \begin{aligned}
\mathrm{i} & =1, \ldots, N \\
\mathrm{~m} & =1, \ldots, L
\end{aligned}
$$

donde: $Z_{i, k}$ es la i-ésima observación de la variable $k$ transformada y $C_{m, k}$ el m-ésimo centro de la misma variable. Si la menor distancia de la observación i es con respecto al centro p, ello implica que i se asignará al estrato con centro p.

Una vez definidos los $L$ estratos iniciales, se calculan las medias de las observaciones asignadas a estos conglomerados, las cuales se considerarán ahora como los nuevos centros. Se estiman entonces las sumas de cuadrados de las observaciones a sus respectivos centros y se verifica cada observación para determinar si un movimiento hacia el centro de otro conglomerado reduce la suma de cuadrados total. Esto último ocurre cuando la reasignación de una observación del estrato $\mathrm{g}$ al h es tal que la distancia al estrato $g\left(d_{g}\right)$ es mayor que la distancia al estrato $\left(d_{g}\right)$ en función de:

$$
\frac{N_{h}}{N_{h}+1} d_{g}^{2}>\frac{N_{g}}{N_{g}+1} d_{h}^{2}
$$

El procedimiento se aplica en forma iterativa hasta que ya no es posible reasignar la observación del estrato $\mathrm{g}$ al estrato $\mathrm{h}$. El resultado final es una estratificación multivariada de límites óptimos.

B. Ponderadores por variable del índice de componentes principales 1970 y 1980

\begin{tabular}{lcc}
\hline & \multicolumn{2}{c}{ Ponderadores } \\
\cline { 3 - 3 } Indicador & 1970 & 1980 \\
\hline PEA con ingresos iguales o mayores al salario mínimo & .7519 & .4792 \\
Alfabetismo de la población de 10 años y más & .8869 & .8907 \\
Población adulta con instrucción & .8767 & .8855 \\
Población adulta con enseñanza media & .8660 & .7938 \\
Viviendas con piso diferente a tierra & .9380 & .9309 \\
Viviendas con agua entubada & .9033 & .8820 \\
Viviendas con tuberías de drenaje & .8430 & .8351 \\
Viviendas con energía eléctrica & .9149 & .9159 \\
Viviendas con más de un cuarto & .7946 & .7528 \\
1 000 menos la tasa bruta de mortalidad & .4079 & .4705 \\
\hline
\end{tabular}

Fuente: Cuadro 6. 


\section{Bibliografía}

Atkinson, A.B. (1970), "On the Measurement of Inequality", Journal of Economic Theory, II.

Dirección General de Estadística (1973), IX Censo General de Población. México, D. F., Secretaría de Industria y Comercio.

Hernández Laos, Enrique (1984), "La Desigualdad Regional en México 1900-1980", en Cordera y Tello (eds.), La Desigualdad en México, México, D. F., Siglo XXI.

Instituto Nacional de Estadística, Geografía e Informática (INEGI) (1985), Sistema de Cuentas Nacionales de México. Estructura Económica Regionai. Producto Interno Bruto por Entidad Federativa, 1970, 1975 y 1980, México, D. F., Secretaría de Programación y Presupuesto (1984), X Censo General de Población y Vivienda. México, D.F., Secretaría de Programación y Presupuesto.

Jarque, Carlos (1981), "A Solution to the Problem of Optimun Stratification in Multivariate Sampling”, Journal of the Royal Statistical Society, 30:2.

Ram R. (1982), "Composite Indices of Physical Quality of Life, Basic Need Fulfillment, and Income: A Principal Component Representation”, Journal of Development Economics, 11.

Scott, Ian (1982), Urban and Spatial Development in Mexico, Baltimore: Johns Hopkins.

Sen, Amartya (1973), On Economic inequality, Oxford: The University Press.

Unikel, Luis y Edmundo Victoria (1970), "Medición de algunos aspectos del desarrollo socioeconómico de las entidades federativas de México, 1940-1960”. Demografía y Economía IV:3, México, D.F., El Colegio de México. 
